\title{
Are commonly-used psychoactive medications associated with lower urinary tract symptoms?
}

\author{
Susan A. Hall, Ph.D. ${ }^{1}$, Nancy N. Maserejian, Sc.D ${ }^{1}$, Carol L. Link, Ph.D. ${ }^{1}$, William D. Steers, \\ M.D., and John B. McKinlay, Ph.D. ${ }^{1}$ \\ ${ }^{1}$ New England Research Institutes, 9 Galen St., Watertown, MA, 02472 USA \\ 2University of Virginia School of Medicine, Charlottesville, VA, 22908, USA
}

\section{Abstract}

Purpose-Lower urinary tract symptoms (LUTS) such as urinary frequency and urgency are bothersome and associated with reduced quality-of-life. Atypical antipsychotics (AAPs) have been implicated in increasing the risk of urinary incontinence. In a large community-based sample of men and women, we examined the associations of AAP use and selective serotonin reuptake inhibitor (SSRIs) use with LUTS.

Methods-Data were collected (2002-2005) from a generalizable sample of Boston, MA, USA residents aged 30-79 $(\mathrm{N}=5503)$. LUTS were assessed using the American Urologic Association Symptom Index (AUA-SI). The prevalence of clinically-significant LUTS was estimated using a cutoff AUA-SI score of $8+$ to indicate moderate-to-severe symptoms. Confounder-adjusted odds ratios (ORs) and 95\% confidence intervals $(\mathrm{CI})$ were calculated from multivariate logistic regression to estimate the associations for psychoactive drugs used in the previous month (SSRIs, AAPs, both) and LUTS.

Results-Among women, AAP users had a higher prevalence of LUTS (46.2\%) compared to SSRI users (23.5\%) and those with depressive symptoms not using SSRIs or AAPs (26.3\%). Corresponding prevalence estimates among men were $32.7 \%, 29.8 \%$, and $33.3 \%$. In multivariate models, AAP use was significantly associated with LUTS among women when used either with SSRIs (OR=2.72, 95\%CI:1.45-5.10), or without SSRIs (OR=3.05, 95\%CI:1.30-7.16) but SSRI use without AAP use was not associated with LUTS, compared to non-users without depressive symptoms. No associations were observed among men.

Conclusions - In our study, AAPs but not SSRIs were associated with increased prevalence of LUTS among women only. Further prospective research is needed to determine time sequence and cause-and-effect.

\section{Keywords}

antipsychotic agents; antidepressive agents; urination disorders; pharmacoepidemiology; epidemiology

\footnotetext{
${ }^{1}$ Corresponding Author: Susan A. Hall, New England Research Institutes, 9 Galen St., Watertown, MA 02472 USA, Tel: 617-923-7747; fax 617-924-0968, shall@neriscience.com.

Conflict of interest statement

Dr. William Steers reports service to the American Urologic Association as the editor of the Journal of Urology, and is a consultant to New England Research Institutes. All other authors declare no conflicts of interest.
} 


\section{Introduction}

Recent market research data indicate that in 2008, antipsychotics surpassed lipid regulators as the top-selling therapeutic class in the U.S.[1] A nationally representative survey of physicians found that the majority (93\%) of antipsychotics prescribed in the U.S. in 2008 were 'second generation' atypical antipsychotics (AAPs), with a marked shift away from first generation 'typical' antipsychotics from previous years.[2] This trend appears to be present internationally as well.[3] In the U.S., patient populations for AAPs have broadened to include children and older adults,[4] and it is estimated that only $40 \%$ of all AAP use is 'on-label'.[2] The recent dramatic changes in the prescribing landscape have been accompanied by criticisms,[5] and have raised concerns about drug safety especially in children,[6] older adults,[7] and among 'new indication' populations such as persons with depression.[8]

Lower urinary tract symptoms (LUTS) such as urinary frequency and urgency are bothersome and associated with reduced quality-of-life.[9] Urinary incontinence is a side effect of antipsychotics;[10] as is urge incontinence [11] while a recent study of clozapine (an AAP) suggested that users suffered from an excess of a broader range of LUTS than incontinence alone.[12] There is also evidence that selective serotonin reuptake inhibitor (SSRI) antidepressants could increase the risk of LUTS.[13] Prior associations between SSRIs and nocturia and risk of urinary incontinence have been documented.[14, 15] Possible pathways include promotion of cholinergic neuromuscular transmission in the detrusor muscle, as well as influence on autonomic function.[16, 17] Furthermore, there is a largely consistent association of depression itself with urologic symptoms in multiple studies of cross-sectional[18-21] or longitudinal[22, 23] design, making it difficult to sort out any underlying effect of depression from indicated medications. We previously found an association between depression and LUTS[24] in our population-based epidemiologic study. The current analyses were conducted in order to further consider the association of psychoactive medications (specifically, SSRIs and AAPs) with LUTS, while accounting for the underlying influence of depression.

\section{Materials and Methods}

\section{Study design and data collection}

The Boston Area Community Health $(\mathrm{BACH})$ Survey is a population-based, epidemiologic study conducted among 5,503 men and women aged 30 to 79 residing in Boston, Massachusetts. A multistage, stratified cluster sampling design was used to recruit approximately equal numbers of persons from pre-specified age $(30-39,40-49,50-59,60$ 79 ), race/ethnicity (black, Hispanic, white), and gender groups. This cross-sectional analysis used baseline data collected 2002-2005 during a two-hour, in-person interview conducted by a trained, bilingual interviewer after acquisition of written informed consent. Interviews for $63.3 \%$ of eligible persons were completed, with a resulting study population of 2301 men and 3202 women (1767 black participants, 1877 Hispanic, 1859 white). All protocols and procedures were approved by the Institutional Review Board of New England Research Institutes. Further details of the study are available.[25]

\section{Urologic symptoms and depression measures}

LUTS were defined using the American Urologic Association (AUA) Symptom Index (AUA-SI) (equivalent to the International Prostate Symptom Score [IPSS] scale) that measures seven urologic symptoms.[26] Higher scores (range 0-35) indicate more symptoms. We defined the presence of LUTS as a score of $\geq 8$, corresponding to moderateto-severe symptoms. For graphical displays, LUTS severity categories were defined using 
the AUA-SI as follows: no symptoms (score=0), mild (1-7), moderate (8-19), severe $(\geq 20)$. We also examined mean storage and voiding storage subscales of the AUA-SI. For storage symptoms, relevant questions were related to frequency, urgency, nocturia, with a possible score range of $0-15$. For voiding symptoms, relevant questions were incomplete emptying, intermittency, weak stream, and hesitancy (possible score range, 0-20). An individual's scores on the subscales sum to the full AUA-SI score. Urologic symptom interference with activities of daily living was assessed using the validated Epstein scale.[27] A score of 0 was assigned for "none of the time" while a score of four was assigned to "all of the time" for seven questions on usual activities (score range, 0-28); higher scores indicate more bother. Depressive symptoms were considered present among participants with at least five of eight symptoms on the abridged Center for Epidemiologic Studies Depression Scale.[28]

\section{Psychoactive medication use}

$\mathrm{BACH}$ participants were asked to gather medications used in the previous four weeks (for the purposes of our survey, considered "current use") for label recording by the interviewer, as well as asked to report medications used for specific indications during the same time period. Medication labels and/or responses were coded by therapeutic class. The main psychoactive drugs of interest for this analysis were 1) antidepressants, and 2) atypical antipsychotics (AAPs) (e.g., risperidone, aripiprazole). The antidepressant group includes selective serotonin reuptake inhibitors (SSRIs) (e.g., fluoxetine), selective serotonin norepinephrine reuptake inhibitors (SNRIs) (e.g., venlafaxine), serotonin modulators (trazadone and nefazodone), and buproprion, but excludes tricyclic antidepressants. As 76\% of the SSRI/SNRI and 'miscellaneous' antidepressant group were using SSRIs, for simplicity they are hereinafter called SSRIs. In this analysis sample, tricyclic antidepressants (TCAs) were used much less frequently than the SSRI group (2.9\% vs. $13.9 \%)$. We did not include TCAs with SSRIs as they are directly prescribed for urinary symptoms and thus any observed association may be due to 'confounding by indication'. However, we examined the coprevalence of TCA use with SSRIs and AAPs, and the resilience of modeling results to adjustment for TCAs. No BACH participants used duloxetine, an SNRI that may improve stress urinary incontinence among women.[29]

\section{Covariates}

Potential covariates were chosen based on prior discovery of associations with LUTS in our survey.[24] A socioeconomic status (SES) variable was constructed as a function of standardized income and education variables for the Northeastern U.S. and reclassified into low, middle and high.[30] Other comorbidities were based on the query, "Have you ever been told by a health care provider that you have or had...?" Cardiovascular disease (CVD) was a composite yes/no variable (see Table 1 notes). Body mass index (BMI) was calculated from interviewer-measured weight and height. Physical activity was defined using the Physical Activity Scale for the Elderly (low 0-99, medium 100-249, high 250+).[31]

\section{Analytic sample and statistical analysis}

Our overall analytic goal was to understand the association between classes of psychoactive medication and LUTS, without confounding by established urologic conditions affecting bladder function. We therefore excluded subjects who self-reported a history of genitourinary cancers, prolapsed bladder, previous surgery affecting the bladder or prostate, surgery for urinary incontinence, or who reported having use of a catheter recommended to them. Persons with Parkinson's disease and multiple sclerosis were also excluded due to the strong influence of these diseases on LUTS. 482 subjects were removed, leaving 5,021 persons ( $91.2 \%$ of the original sample). 
Analyses were conducted using v9.2 of SAS and v10.0.1 of SUDAAN. Missing data were replaced using multiple imputation; $<1 \%$ of data were missing for most variables. Income (used to construct SES) was missing for 3\%, 4\% and 11\% of white, black and Hispanic subjects, respectively. Medication variables were not imputed. Descriptive analyses were weighted for study design, and the characteristics of 4 groups were examined: 1) users of SSRIs only; 2) AAP users; 3) non-users with depressive symptoms; 4) non-users without depressive symptoms. Those who used AAPs as well as SSRIs $(\mathrm{N}=79)$ were placed in the AAP group, as AAP users were much more likely to use SSRIs than SSRI users were to use AAPs, thus our grouping better represents typical patterns of community use.

Multivariate logistic regression modeling was used to identify associations between psychoactive medications and LUTS, using adjusted odds ratios (ORs) and 95\% confidence intervals (CIs). In models, the main exposure variable was constructed to account for overlap and separately estimated ORs for LUTS considering: 1) joint exposure to both SSRIs and AAPs; 2) exposure to AAPS but not SSRIs; 3) exposure to SSRIs but not AAPs; and 4) those using neither (as the common referent category). As study design variables, race/ethnicity and age were always retained in models, as were depressive symptoms in order to control for confounding by indication and severity (given some drug-exposed persons were still reporting depressive symptoms). Additional potential confounders considered for models included: arthritis, BMI, CVD, diabetes (type I or II), high blood pressure, physical activity, SES, use of statins and antihypertensives. To determine the structure of the final model (Model 2), a full model was fit and using backward selection, only those covariates that induced a 15\% change in the OR at any level of the main exposure, or were significantly associated with the outcome (Wald $\mathrm{F}$ test $\mathrm{p}<0.10$ ) were included.

\section{Results}

Approximately $40 \%$ and $50 \%$ of current users of SSRIs only and AAPs, respectively, met our definition of having depressive symptoms (Table 1). SSRI users had the oldest mean age of the four groups $(49.9 \mathrm{y})$, while AAP users had the youngest (45.7y). Considering all four groups, the highest proportion of white subjects was observed among SSRI users (77.2\%) while the highest proportions of black and Hispanic subjects (41.5\% and 23.5\%, respectively) were always observed in the group who reported depressive symptoms and were not users of SSRIs or AAPs. The proportion of high SES persons was greatest in the no drug use/no depressive symptoms group, followed by the SSRI group (28.3\% and 25.4\%, respectively). Among AAP users, only $1.4 \%$ were high SES. Except for high blood pressure, there was a general pattern of higher comorbidities and obese BMI among the three groups with either medication use or untreated depressive symptoms compared to those without; differences were especially marked for CVD and diabetes. 49.1\% of AAP users also used SSRIs, while a small proportion of all groups (highest, $6.7 \%$ among SSRI users) used TCAs.

Figures 1A and 1B depict the prevalence of LUTS severity categories among the four groups, by gender. Among men (Figure 1A), 97\% of AAP users reported LUTS of any severity compared to $78.1 \%$ of SSRI users. The prevalence of moderate-to-severe symptoms (AUA-SI score $>8$ ), however, was approximately equal across the SSRI, AAP and depressive symptom groups $(29.8 \%, 32.7 \%$ and $33.3 \%$, respectively). Among women (Figure 1B), most of the SSRI and AAP users (92.2\% and 99.8\%, respectively) reported at least mild LUTS, but the prevalence of moderate-to-severe LUTS was much higher in the AAP group $(46.2 \%)$ compared to any other group (next highest: untreated depression, $26.3 \%$ ). Considering overall AUA-SI mean scores, AAP users consistently had the highest scores, although differences were stronger for women (7.8) than men (6.2) (Figure 2A). Among men and women, AUA-SI scores were similar considering SSRI users and those 
with untreated depression. Mean storage scores were always higher than voiding scores in all groups and generally higher among women than men, but among women, the highest voiding and storage scores were observed in the group using AAPs. Finally, mean urologic symptom interference scores among women were markedly higher among AAP users (7.8) compared to SSRI users (3.2) and those with depressive symptoms without use of these medications (3.1) (Figure 2B), suggesting that women AAP users are more bothered by symptoms. Among men, scores were not markedly different between the AAP group and the untreated depression group (2.4 and 2.2, respectively) and were lowest among SSRI users (1.2).

We further considered whether AUA scores were different by depression status, among psychoactive medication users (data not shown in figure). Mean AUA scores among SSRI users were always higher among those with concurrent depressive symptoms (men: 7.0 with depressive symptoms, 4.6 without; women: 6.2 with depressive symptoms, 5.0 without). Differences in mean scores were of similar magnitude for male AAP users (men: 7.0 with depressive symptoms, 5.7 without) but female AAP users had greater differences in mean scores by depression status ( 9.4 with depressive symptoms, 5.6 without). We subsequently tested for statistical interaction between psychoactive drug use and depression status in models for LUTS, but found no significant interactions (all $\mathrm{p}>0.22$ ) suggesting that the association of psychoactive medications and LUTS did not differ by depression status.

Multivariate logistic regression models for prevalent LUTS are presented in Table 2. Among men, there were no significant associations observed for use of AAPs with SSRIs, use of AAPs without SSRIs, or use of SSRIs without AAPs, following adjustment for age, race/ ethnicity and depressive symptoms (Model 1) or further covariate adjustment (Model 2). While use of SSRI antidepressants and AAPs were not associated with LUTS among men, depression was strongly and significantly associated (Model 2 OR=3.17, 95\% CI: $2.35-$ 4.27). Among women, however, there were significant associations for all three drug groupings in the minimally-adjusted model (Model 1). After further adjustment (Model 2), joint use of AAPs and SSRIs, and AAPuse (but not SSRIuse) had persistent, significant associations with LUTS. The observed associations for joint AAP and SSRIs use or AAPs without SSRIs had imprecise confidence intervals but were of strong magnitude (joint use: $\mathrm{OR}=2.72$, 95\% CI 1.45-5.10; AAP use only: OR=3.05, 95\% CI: 1.30-7.16) suggesting that AAP users have a higher prevalence of LUTS after multivariate adjustment, and the 'jointuse' association is driven by use of AAPs. The magnitude of these associations exceeded that of depression in the model (OR for depression=1.72, 95\% CI: 1.38-2.15).

We further examined whether confounding by TCA use explained the associations between AAP use and LUTS among women. Adjustment for use of these drugs changed the ORs minimally $(<10 \%)$ and did not attenuate the previously-observed associations. Among women, to examine whether confounding by depression could still be playing a role, we ran additional models among a subsample of only those who had depression symptoms. Although the smaller sample size reduced the precision of confidence intervals, the finding of significant positive associations with LUTS for AAP use with and without SRRI use was persistent. We also ran our final models among women considering an exposure group containing only SSRIs, rather than SSRI/SNRIs/miscellaneous antidepressants, and found our results largely consistent, with changes in magnitude of ORs $<15 \%$ and no changes in statistical significance (data not shown). Similarly among men, while we had insufficient numbers to model a joint exposure category for 'pure' SSRIs and AAPs, our estimate for use of 'pure' SSRIs without AAPs was similar to the estimate for use of SSRI/SNRI/ miscellaneous antidepressants without AAPs. 


\section{Discussion}

In a population-based study, we examined the characteristics of users, prevalence of LUTS, symptom severity, and urinary symptom interference scores considering current users of popular psychoactive medications (SSRIs including SNRIs, and AAPs) and non-users with and without depressive symptoms, and used multivariate methods to estimate adjusted associations for drug use and LUTS. Our results were consistent with respect to women users of AAPs having the highest overall prevalence of LUTS of any severity, the highest prevalence of moderate-to-severe symptoms, and the greatest symptom interference scores. The elevation in voiding scores suggested that the excess of symptoms in this group was not merely explained by storage alone. We found the excess LUTS persisted after risk factor adjustment, resulting in substantial and statistically significant positive associations of AAP use (with and without SSRIs) with LUTS among women. Our cross-sectional analysis measures correlation/association and cannot determine cause and effect. A prospective analysis of this research question is planned among persons participating in a second phase of BACH data collection.

The reasons for our observed gender differences for AAPs are not known. Antipsychotics are lipophilic drugs likely to be metabolized differently among women compared to men due to differences in body composition, with a resulting higher volume distribution and higher serum levels at similar doses to men;[32] [33] female users of antipsychotics have also been found to have greater incidence of adverse events compared to males.[34] However, SSRIs are also lipophilic and the same gender difference in prevalent LUTS was not observed. It is also possible that women in our study using antipsychotics may differentially report symptoms compared to women taking only SSRIs, but the fact that we did not observe the same for men using antipsychotics suggests otherwise. In a previous clinical study of LUTS measured using an adapted version of the IPSS scale among 101 clozapine users, women had only non-significantly higher mean scores compared to men (7.78 and 7.18, respectively).[12] Because AAPs are not currently indicated as first-line therapy for depression, it is reasonable to speculate that women using AAPs alone had more severe, potentially uncontrolled depression even after adjustment for residual depressive symptoms. However, we observed similar associations comparing joint users of SSRIs and AAPs to AAP-only users, suggesting that 'confounding by severity' did not explain the association.

Among both genders, depression had a significant association with LUTS in multivariate models adjusted for the use of antidepressants. This finding is consistent with many previous studies.[18-23] In our study, the association for depressive symptoms was stronger among men compared to women. While the reasons for this are unknown, the gender difference has also been observed in the BACH Survey for urinary incontinence[35] and in the large EPIC study for the symptoms of overactive bladder with incontinence.[36] A qualitative study of $\mathrm{BACH}$ participants found that men as well as women felt that urologic symptoms including frequency and urgency were stigmatizing, and that the nature of the stigma was different than for women.[37] This in turn suggests that the psychological impact of symptoms may also differ by gender.

We did not confirm an association between SSRI use and LUTS in either gender. A recent large study by Wuerstle et. al found a significant association of antidepressant use with LUTS among men aged 45 to 69 years $(\mathrm{OR}=1.36,1.29-1.44)$.[13] This result (adjusted for BPH status) was similar in magnitude to our adjusted findings for men for SSRIs in Table 2, but was not further adjusted for comorbidities such as depressive symptoms and diabetes which may explain the differences compared to our results. When minimally adjusted for age and race/ethnicity, our association for use of SSRIs without AAPs among men was also statistically significant (OR=1.98, 95\% CI: 1.36-2.87, data not shown). In their analysis, 
Wuerstle et. al combined all antidepressants together, which may further explain differences between our results. Other studies of SSRIs and related LUTS have included nocturia (a symptom on the AUA/IPSS scale). A study of Swedish men and women found that both depression and SSRI use were significantly associated with nocturia (OR for SSRI use $=2.2$, 95\% CI 1.1-4.5).[14] However, as with Wuerstle, the analysis was unadjusted for the influence of comorbid risk factors.. Another study of nocturia conducted in Finland found antidepressants positively associated with nocturia $(\mathrm{OR}=3.16,95 \% \mathrm{CI}: 1.29-7.73)$ after multivariate adjustment, but only among men.[38] The subtypes of antidepressants studied were not defined. Finally, a recent longitudinal analysis found that men with depression at baseline were more likely to develop moderate to severe nocturia five years later, with an effect apparent only in those whose depression was not drug-treated; antipsychotics were also not associated with incident nocturia. These results lend some support the findings of our study by suggesting that use of psychoactive medications does not cause incident nocturia among men.[23]

Despite this population-based evidence, however, there are case reports of urinary disturbances associated with SSRI use,[39] suggesting that unique effects may occur on an individual level. Each SSRI has a unique pharmacologic profile and this could explain the opposing actions on voiding behavior reported in the literature ranging from urinary incontinence to retention.[15] Our analysis combined all SSRIs and potentially missed opposing drug effects. In addition to the cross-sectional analysis, our study is limited by a small number of exclusive users of AAPs. However, our grouping of AAP use with SSRI users may best represent patterns of 'real world' drug use. Our modeling results suggested that the chosen groupings in Table 1 were supported, considering that AAP use appeared to confer the association among women, whether with or without SSRIs. We did not consider dose or duration of use, and risk of adverse urologic effects may vary by these factors. There is also the potential for misclassification of depression, as severity may vary over time. Strengths of our study include inclusion of both genders, the ability to consider overlap with other medications, and the ability to adjust for a wide variety of known risk factors for LUTS.

Our results provide some evidence that SSRIs are not associated with an increased prevalence of LUTS in the community. As our gender-specific results for the association of AAP use with increased prevalence of LUTS among women are novel, these findings should be further investigated.

\section{Acknowledgments}

The authors thank Ray S. Rosen, Mary P. Fitzgerald, Deborah C. Brander and Gavin Miyasato for contributions to this manuscript.

\section{References}

1. Kuehn BM. Questionable antipsychotic prescribing remains common, despite serious risks. Jama. 303(16):1582-1584. [PubMed: 20424239]

2. Alexander GC, Gallagher SA, Mascola A, Moloney RM, Stafford RS. Increasing off-label use of antipsychotic medications in the United States, 1995-2008. Pharmacoepidemiol Drug Saf. 2011; 20(2):177-184. [PubMed: 21254289]

3. Verdoux H, Tournier M, Begaud B. Antipsychotic prescribing trends: a review of pharmacoepidemiological studies. Acta psychiatrica Scandinavica. 2010; 121(1):4-10. [PubMed: 20059452]

4. Crystal S, Olfson M, Huang C, Pincus H, Gerhard T. Broadened use of atypical antipsychotics: safety, effectiveness, and policy challenges. Health Aff (Millwood). 2009; (5):w770-w781. [PubMed: 19622537] 
5. Tyrer P, Kendall T. The spurious advance of antipsychotic drug therapy. Lancet. 2009; 373(9657): 4-5. [PubMed: 19058841]

6. McKinney C, Renk K. Atypical antipsychotic medications in the management of disruptive behaviors in children: safety guidelines and recommendations. Clinical psychology review. 2010; 31(3):465-471. [PubMed: 21130552]

7. Ballard C, Creese B, Corbett A, Aarsland D. Atypical antipsychotics for the treatment of behavioral and psychological symptoms in dementia, with a particular focus on longer term outcomes and mortality. Expert Opin Drug Saf. 2011; 10(1):35-43. [PubMed: 20684745]

8. DeBattista C, DeBattista K. Safety considerations of the use of second generation antipsychotics in the treatment of major depression: extrapyramidal and metabolic side effects. Current drug safety. 2010; 5(3):263-266. [PubMed: 20394571]

9. Litman HJ, McKinlay JB. The future magnitude of urological symptoms in the USA: projections using the Boston Area Community Health survey. BJU Int. 2007; 100(4):820-825. Erratum in: BJU Int. 2007 Oct;2100(2004):2971. [PubMed: 17550412]

10. Schneider LS, Dagerman K, Insel PS. Efficacy and adverse effects of atypical antipsychotics for dementia: meta-analysis of randomized, placebo-controlled trials. Am J Geriatr Psychiatry. 2006; 14(3):191-210. [PubMed: 16505124]

11. Bonney WW, Gupta S, Hunter DR, Arndt S. Bladder dysfunction in schizophrenia. Schizophrenia research. 1997; 25(3):243-249. [PubMed: 9264179]

12. Jeong SH, Kim JH, Ahn YM, Lee KY, Kim SW, Jung DC, Kim YS. A 2-year prospective followup study of lower urinary tract symptoms in patients treated with clozapine. Journal of clinical psychopharmacology. 2008; 28(6):618-624. [PubMed: 19011429]

13. Wuerstle MC, Van Den Eeden SK, Poon KT, Quinn VP, Hollingsworth JM, Loo RK, Jacobsen SJ. Contribution of common medications to lower urinary tract symptoms in men [letter]. Arch Intern Med. 2011; 171(18):1680-1682. [PubMed: 21987200]

14. Asplund R, Johansson S, Henriksson S, Isacsson G. Nocturia, depression and antidepressant medication. BJU Int. 2005; 95(6):820-823. [PubMed: 15794790]

15. Movig KL, Leufkens HG, Belitser SV, Lenderink AW, Egberts AC. Selective serotonin reuptake inhibitor-induced urinary incontinence. Pharmacoepidemiol Drug Saf. 2002; 11(4):271-279. [PubMed: 12138594]

16. Tonini M, Candura SM. 5-HT4 receptor agonists and bladder disorders. Trends Pharmacol Sci. 1996; 17(9):314-316. [PubMed: 8885694]

17. Bar KJ, Greiner W, Jochum T, Friedrich M, Wagner G, Sauer H. The influence of major depression and its treatment on heart rate variability and pupillary light reflex parameters. Journal of affective disorders. 2004; 82(2):245-252. [PubMed: 15488253]

18. Holden CA, McLachlan RI, Pitts M, Cumming R, Wittert G, Ehsani JP, de Kretser DM, Handelsman DJ. Determinants of male reproductive health disorders: the Men in Australia Telephone Survey (MATeS). BMC public health. 10:96. [PubMed: 20181284]

19. Coyne KS, Kaplan SA, Chapple CR, Sexton CC, Kopp ZS, Bush EN, Aiyer LP. Risk factors and comorbid conditions associated with lower urinary tract symptoms: EpiLUTS. BJU Int 103 Suppl. 2009; 3:24-32.

20. Nuotio M, Tammela TL, Luukkaala T, Jylha M. Association of urgency symptoms with self-rated health, mood and functioning in an older population. Aging Clin Exp Res. 2007; 19(6):465-471. [PubMed: 18172368]

21. Coyne KS, Sexton CC, Irwin DE, Kopp ZS, Kelleher CJ, Milsom I. The impact of overactive bladder, incontinence and other lower urinary tract symptoms on quality of life, work productivity, sexuality and emotional well-being in men and women: results from the EPIC study. BJU Int. 2008; 101(11):1388-1395. [PubMed: 18454794]

22. Wong SY, Woo J, Leung JC, Leung PC. Depressive symptoms and lifestyle factors as risk factors of lower urinary tract symptoms in Southern Chinese men: a prospective study. Aging Male. 13(2):113-119. [PubMed: 20429720]

23. Hakkinen JT, Shiri R, Koskimaki J, Tammela TL, Auvinen A, Hakama M. Depressive symptoms increase the incidence of nocturia: Tampere Aging Male Urologic Study (TAMUS). J Urol. 2008; 179(5):1897-1901. [PubMed: 18353384] 
24. Litman HJ, Steers WD, Wei JT, Kupelian V, Link CL, McKinlay JB. Relationship of lifestyle and clinical factors to lower urinary tract symptoms: results from Boston Area Community Health survey. Urology. 2007; 70(5):916-921. [PubMed: 17919693]

25. McKinlay JB, Link CL. Measuring the Urologic Iceberg: Design and Implementation of the Boston Area Community Health (BACH) Survey. Eur Urol. 2007; 52(2):389-396. [PubMed: 17383808]

26. Barry MJ, Fowler FJ Jr, O'Leary MP, Bruskewitz RC, Holtgrewe HL, Mebust WK, Cockett AT. The American Urological Association symptom index for benign prostatic hyperplasia. The Measurement Committee of the American Urological Association. J Urol. 1992; 148(5):1549_ 1557. discussion 1564. [PubMed: 1279218]

27. Epstein RS, Deverka PA, Chute CG, Panser L, Oesterling JE, Lieber MM, Schwartz S, Patrick D. Validation of a new quality of life questionnaire for benign prostatic hyperplasia. J Clin Epidemiol. 1992; 45(12):1431-1445. [PubMed: 1281223]

28. Turvey CL, Wallace RB, Herzog R. A revised CES-D measure of depressive symptoms and a DSM-based measure of major depressive episodes in the elderly. Int Psychogeriatr. 1999; 11(2): 139-148. [PubMed: 11475428]

29. Mariappan P, Alhasso A, Ballantyne Z, Grant A, N'Dow J. Duloxetine, a serotonin and noradrenaline reuptake inhibitor (SNRI) for the treatment of stress urinary incontinence: a systematic review. Eur Urol. 2007; 51(1):67-74. [PubMed: 17014950]

30. Green LW. Manual for scoring socioeconomic status for research on health behavior. Public Health Rep. 1970; 85(9):815-827. [PubMed: 4989476]

31. Washburn RA, Smith KW, Jette AM, Janney CA. The Physical Activity Scale for the Elderly (PASE): development and evaluation. J Clin Epidemiol. 1993; 46(2):153-162. [PubMed: 8437031]

32. Seeman MV. Gender differences in the prescribing of antipsychotic drugs. Am J Psychiatry. 2004; 161(8):1324-1333. [PubMed: 15285956]

33. Pollock BG. Gender differences in psychotropic drug metabolism. Psychopharmacology bulletin. 1997; 33(2):235-241. [PubMed: 9230636]

34. Aichhorn W, Gasser EM, Weiss C, Adlassnig J, Marksteiner J. Gender differences in parhmacokinetics and side effects of second generation antipsychotic drugs. Current neuropharmacology. 2005; 3:73-85.

35. Tennstedt SL, Link CL, Steers WD, McKinlay JB. Prevalence of and risk factors for urine leakage in a racially and ethnically diverse population of adults: the Boston Area Community Health (BACH) Survey. Am J Epidemiol. 2008; 167(4):390-399. [PubMed: 18182376]

36. Irwin DE, Milsom I, Kopp Z, Abrams P, Cardozo L. Impact of overactive bladder symptoms on employment, social interactions and emotional well-being in six European countries. BJU Int. 2006; 97(1):96-100. [PubMed: 16336336]

37. Elstad EA, Taubenberger SP, Botelho EM, Tennstedt SL. Beyond incontinence: the stigma of other urinary symptoms. Journal of advanced nursing. 2010; 66(11):2460-2470. [PubMed: 20735505]

38. Tikkinen KA, Auvinen A, Johnson TM 2nd, Weiss JP, Keranen T, Tiitinen A, Polo O, Partinen M, Tammela TL. A systematic evaluation of factors associated with nocturia--the population-based FINNO study. Am J Epidemiol. 2009; 170(3):361-368. [PubMed: 19515794]

39. Lowenstein L, Mueller ER, Sharma S, FitzGerald MP. Urinary hesitancy and retention during treatment with sertraline. Int Urogynecol J Pelvic Floor Dysfunct. 2007; 18(7):827-829. [PubMed: 17089079] 
A

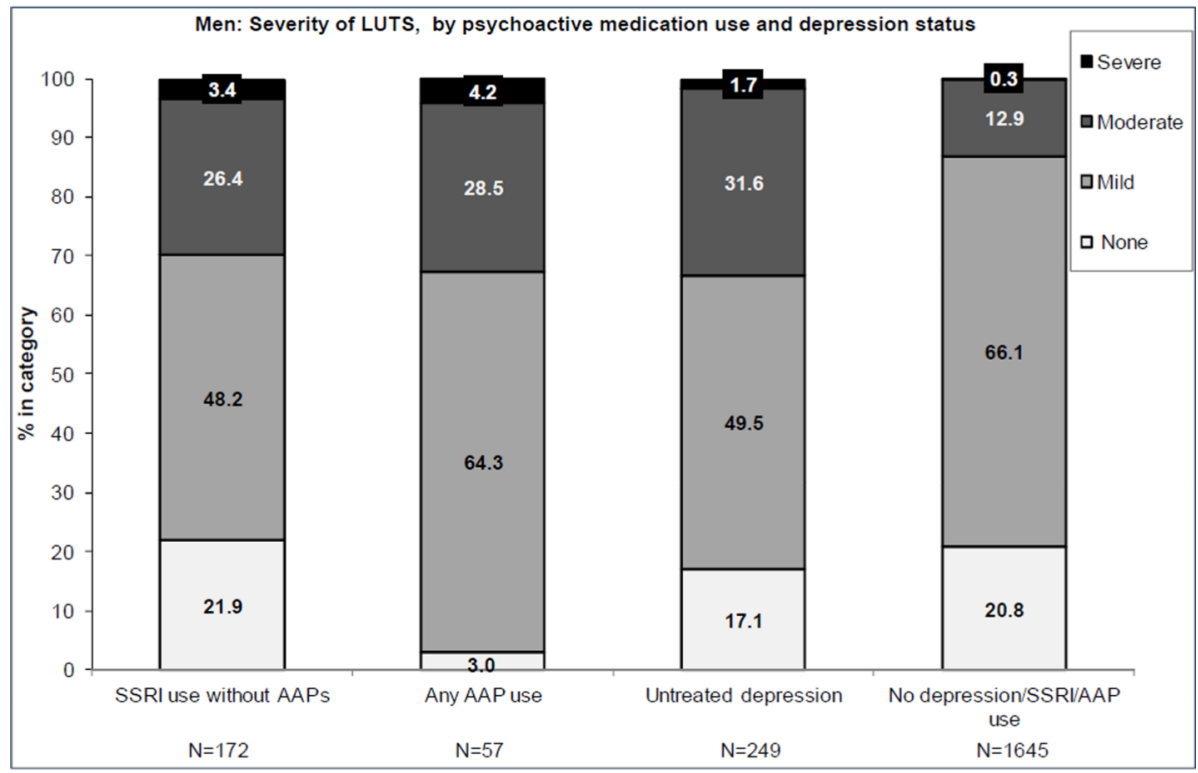

B

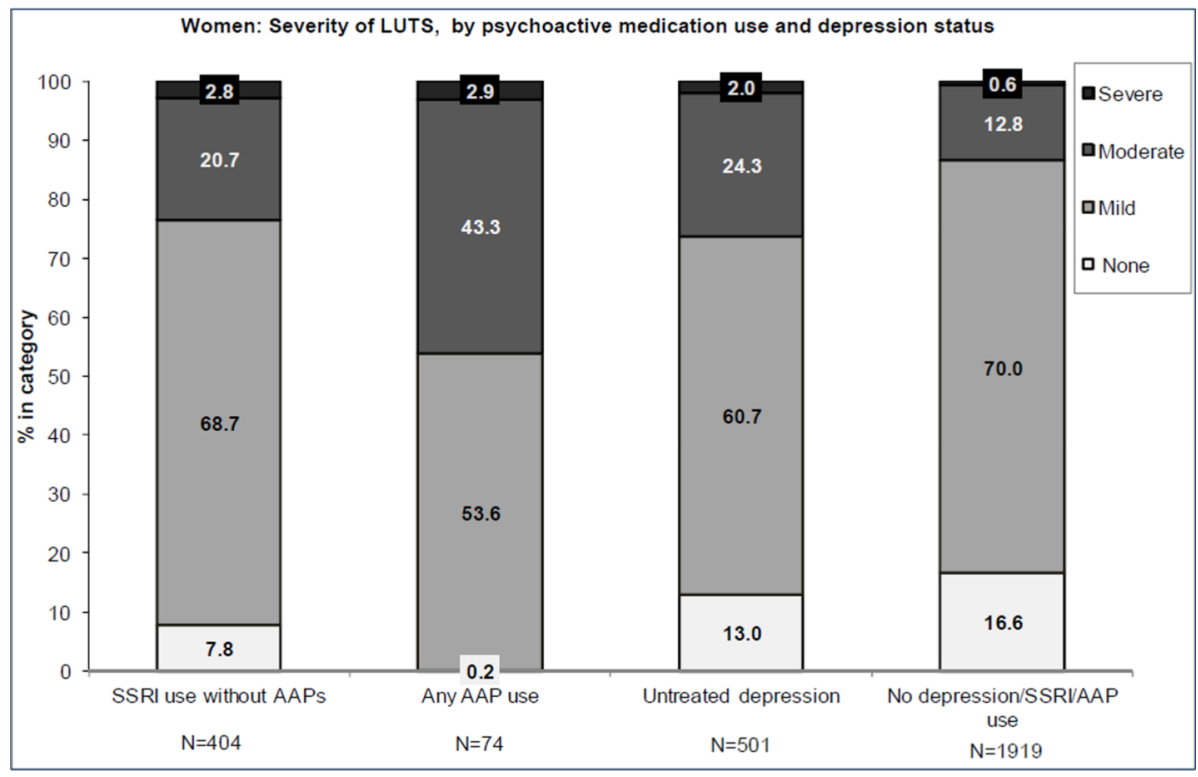

Figure 1.

A. Severity of LUTS among men, by SSRI or AAP medication use and depression status. Untreated depression was defined as depressive symptoms without use of SSRIs or AAPs. The SSRI group also contains SNRIs, serotonin modulators, and buproprion.

B. Severity of LUTS among women, by SSRI or AAP use and depression status. Untreated depression was defined as depressive symptoms without use of SSRIs or AAPs. The SSRI group also contains SNRIs, serotonin modulators, and buproprion. 
A

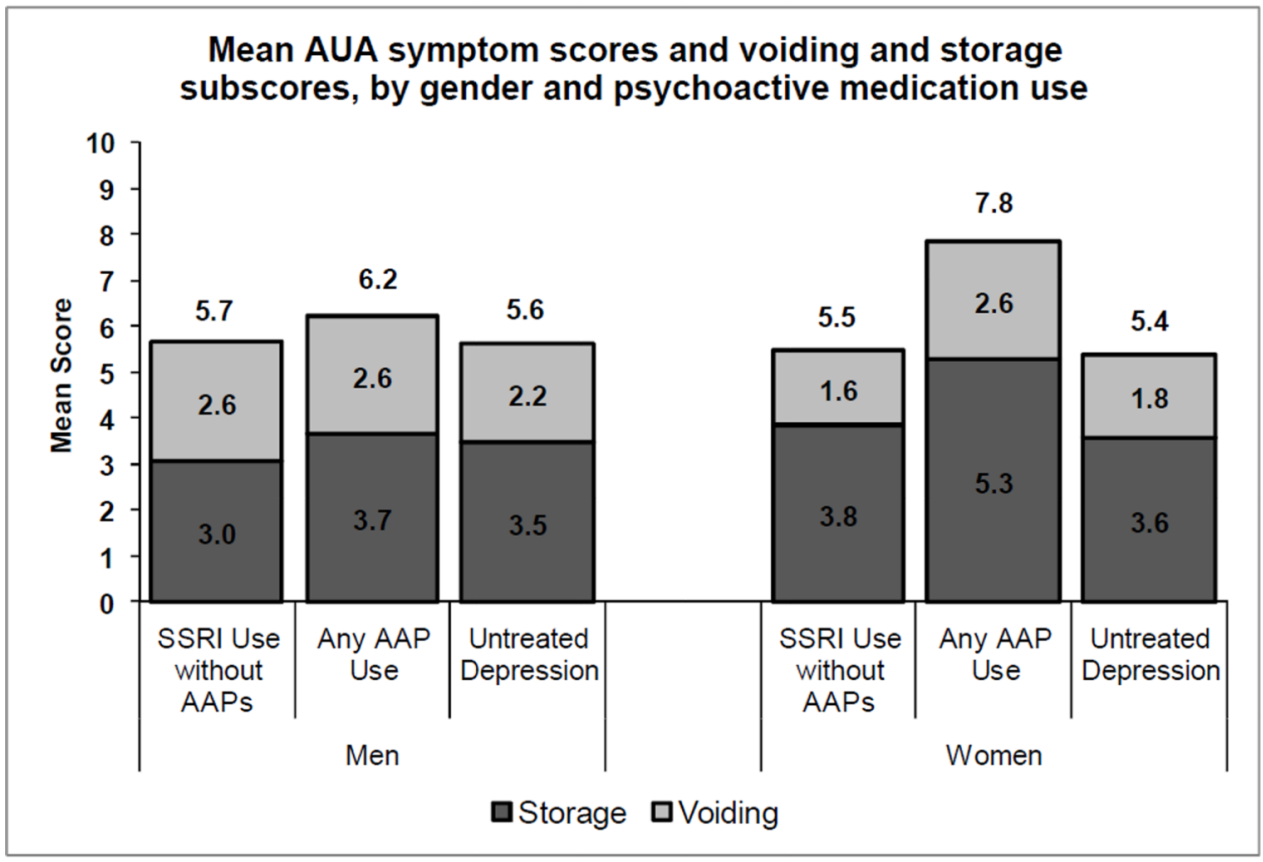

B

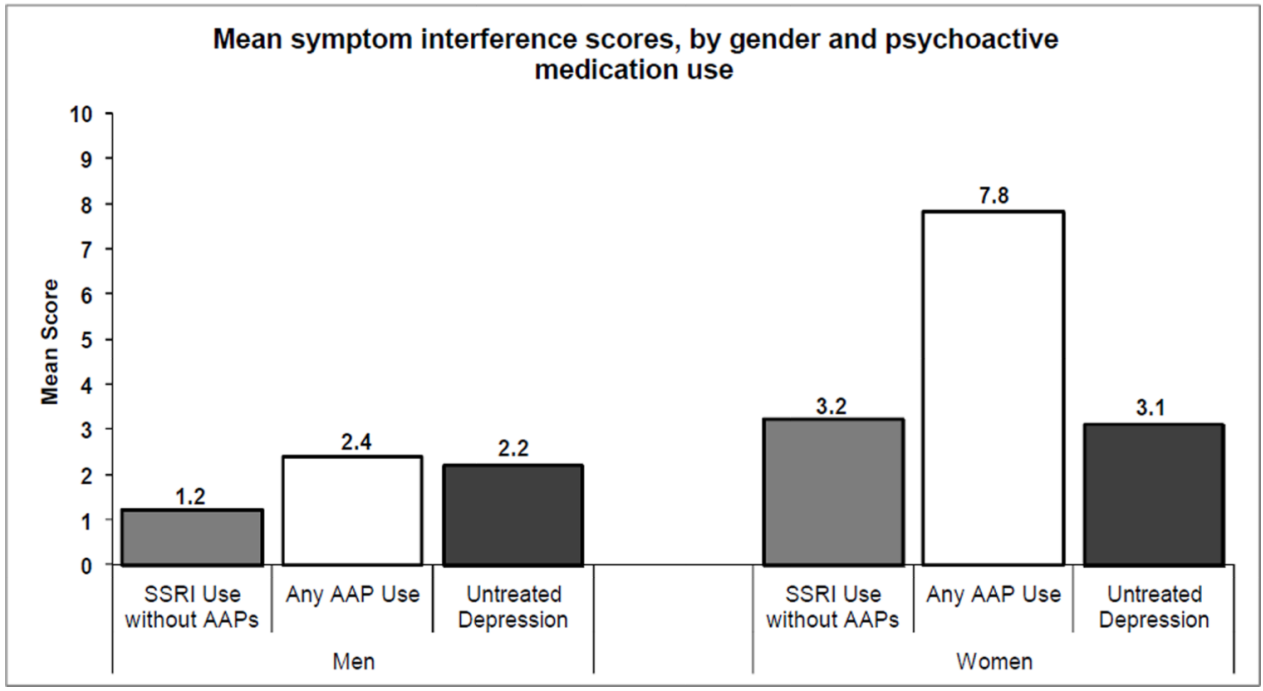

Figure 2.

A. Mean AUA-SI symptom scores and voiding and storage subscores, by gender, SSRI or AAP medication use, and untreated depression (symptoms of depression without use of SSRIs or AAPs). Higher scores indicate more symptoms. Mean scores for those who were not users of these medications and who did not have depressive symptoms were 3.3 and 3.6, for men and women, respectively. The SSRI group also contains SNRIs, serotonin modulators, and buproprion.

B. Mean symptom interference scores, by gender, SSRI or AAP medication use, and untreated depression (symptoms of depression without use of SSRIs or AAPs). Higher scores indicate more symptom interference with activities of daily living. Mean scores for 
those who were not users of these medications and who did not have depressive symptoms were 1.0 and 1.4, for men and women, respectively. The SSRI group also contains SNRIs, serotonin modulators, and buproprion. 


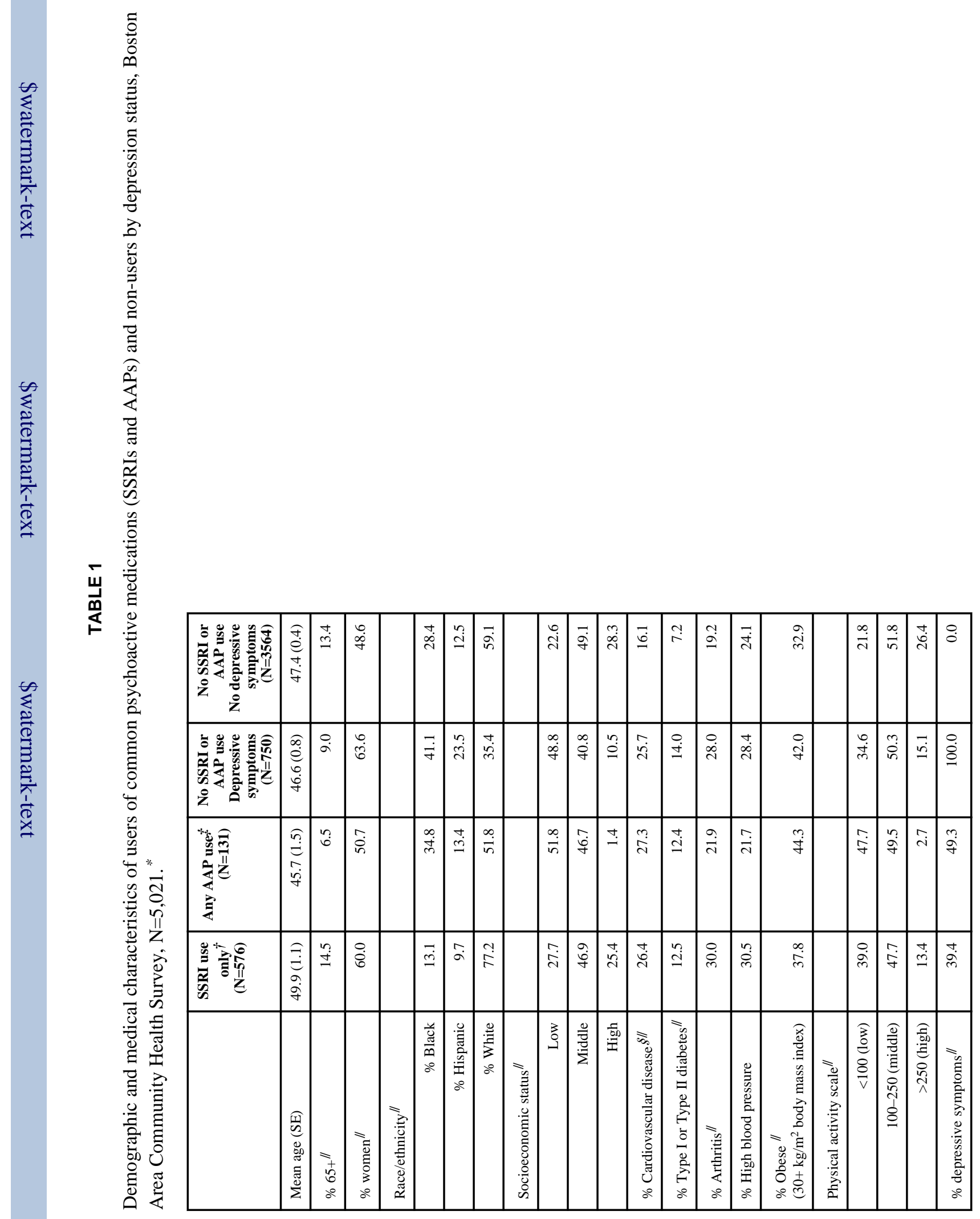




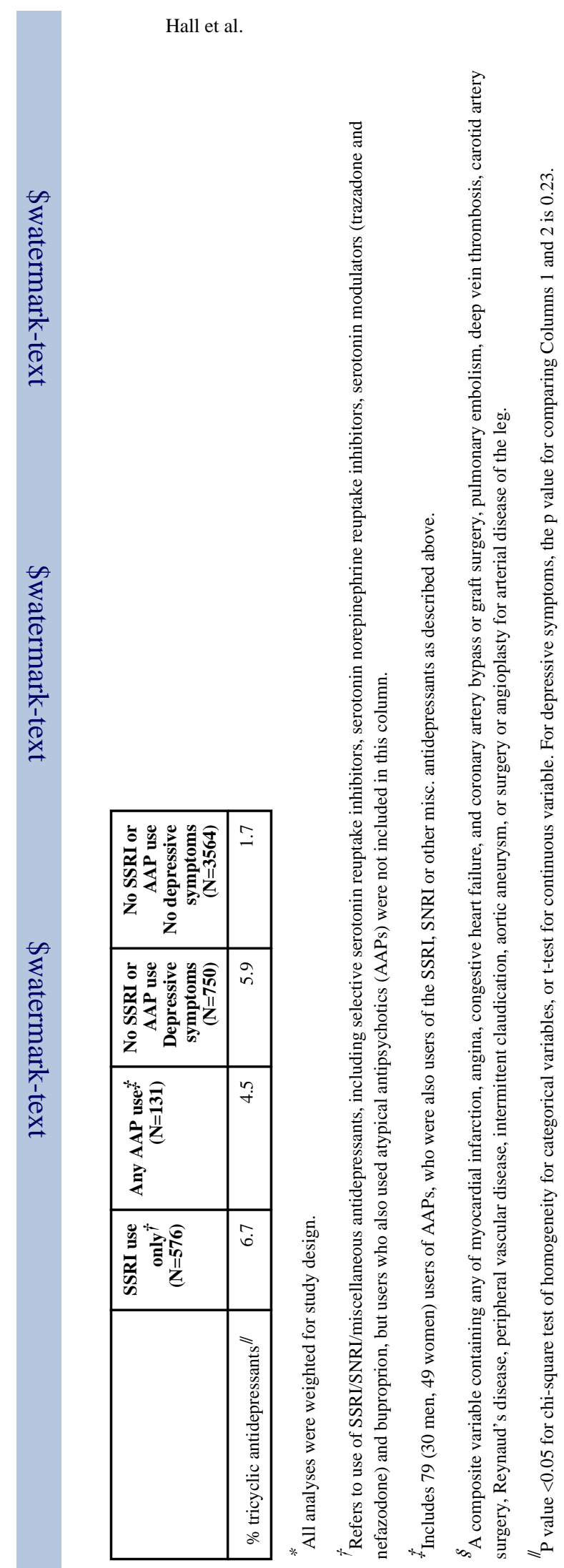

Eur J Clin Pharmacol. Author manuscript; available in PMC 2013 May 01. 


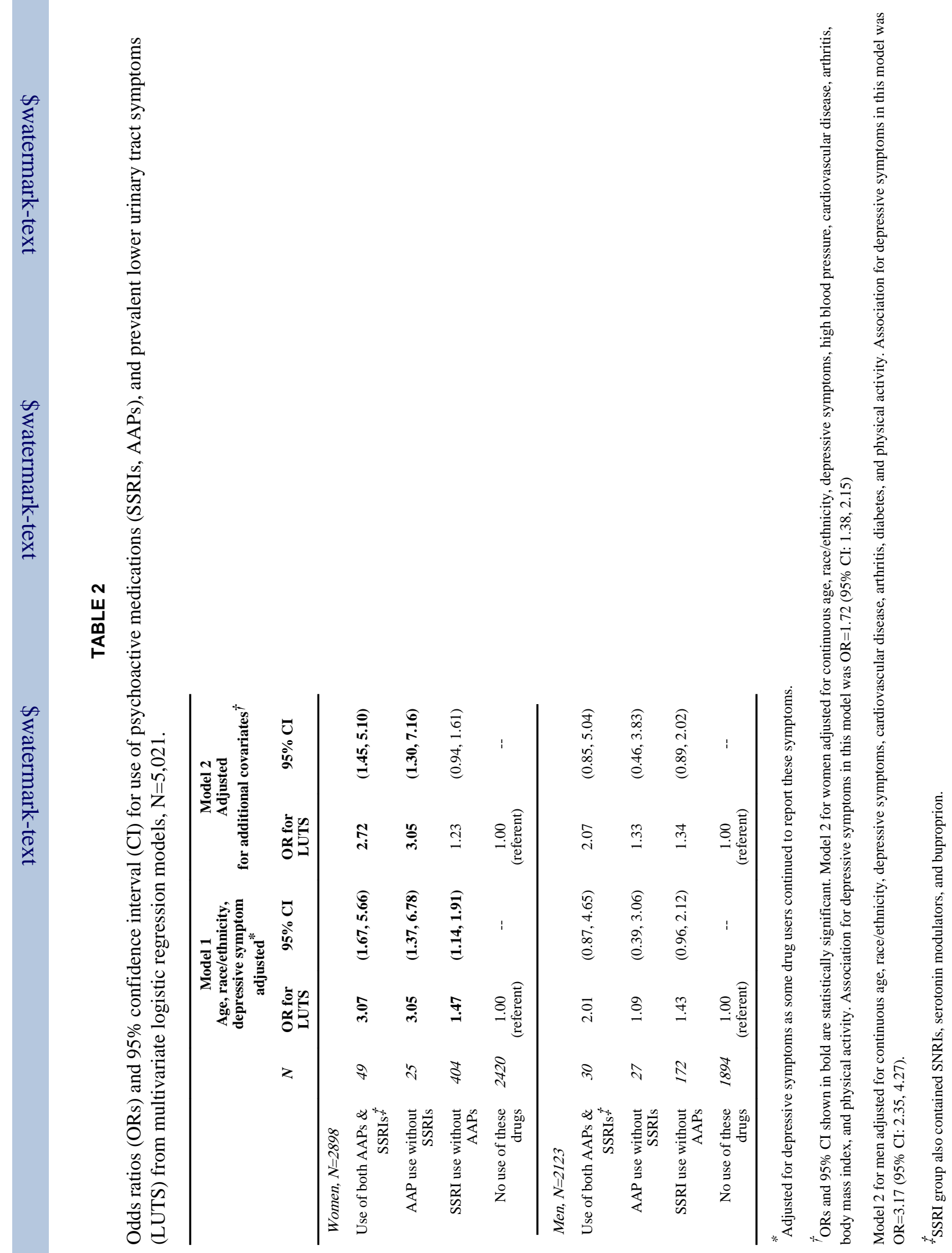

